\title{
Effect of Intra- versus Intermolecular Cross-Linking on the Supramolecular Folding of a Polymer Chain
}

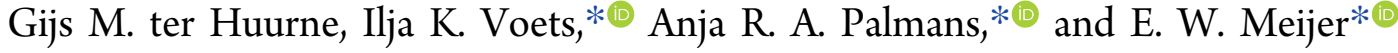

Institute for Complex Molecular Systems, Laboratory of Macromolecular and Organic Chemistry, Eindhoven University of Technology, P.O. Box 513, 5600 MB, Eindhoven, The Netherlands

\section{Supporting Information}

ABSTRACT: Anfinsen's famous experiment showed that the restoration of catalytic activity of a completely unfolded ribonuclease $\mathrm{A}$ is only possible when the correct order of events is followed during the refolding process. Inspired by this work, the effect of structural constraints induced by covalent cross-links on the folding of a synthetic polymer chain via hydrogen-bonding interactions is investigated. Hereto, methacrylate-based monomers comprising either benzene-1,3,5tricarboxamide (BTA)-based or coumarin-based pendants are copolymerized with $n$-butyl methacrylate in various ratios via

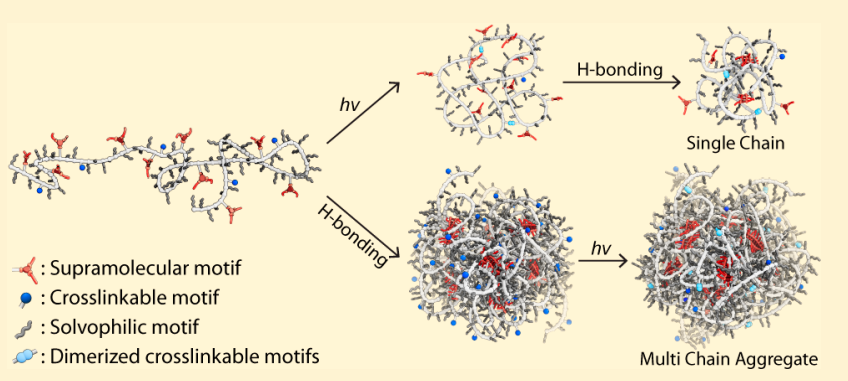
reversible addition-fragmentation chain-transfer (RAFT) polymerization. To assess whether the folding and single-chain polymeric nanoparticle (SCPN) formation depend on the order of events, we compare two folding pathways. In the one case, we first covalently cross-link the coumarin pendants within the polymers in a solvent that prevents hydrogen bonding, after which hydrogen bonding is activated, inducing folding of the polymer. In the other case, we induce hydrogen-bonding interactions between tethered BTAs prior to covalent cross-linking of the coumarin pendants. A combination of circular dichroism (CD) spectroscopy, UV-vis spectroscopy, size-exclusion chromatography (SEC), and dynamic light scattering (DLS) is employed to understand the effect of the structural constraints on the folding behavior of these synthetic polymers. The results show that like in ribonuclease A, the order of events matters greatly and determines the outcome. Importantly, a hydrogen-bond-promoting solvent prevents the formation of SCPNs upon covalent cross-linking and results in multichain aggregates. In contrast, covalently cross-linking the polymer when no hydrogen bonds are present followed by inducing hydrogen bonding favors the formation of SCPNs above the UCST of the methacrylate-based polymer. To our surprise, the two systems show a fundamentally different response to changes in temperature, indicating that also in synthetic polymers differences in the folding pathway induce differences in the properties of the resultant nanostructures.

\section{INTRODUCTION}

In the past decades, the self-assembly of small molecules into supramolecular structures has received significant attention. Today, a wide variety of self-assembling motifs is known that assemble into diverse structures via either isodesmic, cooperative, or even living mechanisms. ${ }^{1-7}$ Recently, research groups started to bridge the field of small molecule selfassembly and the field of controlled polymerization techniques with the aim to fold synthetic polymers into protein-like, structured nanoparticles. $^{8-10}$ In these novel but complex constructs, the self-assembly of the supramolecular grafts is utilized to fold the polymer chains into nanoparticles that comprise a structured interior. Although the conformational mobility of synthetic polymer backbones may cause steric constraints that affect the self-assembly of the grafted supramolecular moieties, recent research has shown that the supramolecular grafts are still capable to self-assemble into their preferred supramolecular assemblies. ${ }^{11,12,21-25,13-20}$

Anfinsen's famous experiment showed that the restoration of catalytic activity of a completely unfolded ribonuclease $A$ is only possible when the correct order of events is followed during the refolding process. ${ }^{26}$ This experiment made us wonder to what extent the presence of structural constraints will affect the hydrogen-bonding-induced folding of synthetic polymers. In ribonuclease A, complete unfolding is achieved by using urea to break up the hydrogen bonds in combination with 2-mercaptoethanol, which is needed to break the covalent disulfide bridges. Upon removal of urea, the formation of specific stabilizing hydrogen bonds folds the polypeptide into its near-native state. This directs the preorganization of the cysteine residues and facilitates the formation of the original disulfide bridges upon the removal of the 2-mercaptoethanol. As a result, the protein is refolded back into its native state and fully regains its catalytic activity. However, when inverting the order of removal by first removing 2-mercaptoethanol, new, non-native disulfide bonds are formed while the polypeptide is still in a disordered state. This results in the formation of disulfide bridges between incorrect cysteine residues, prevent-

Received: July 30, 2018

Revised: September 28, 2018

Published: October 29, 2018 


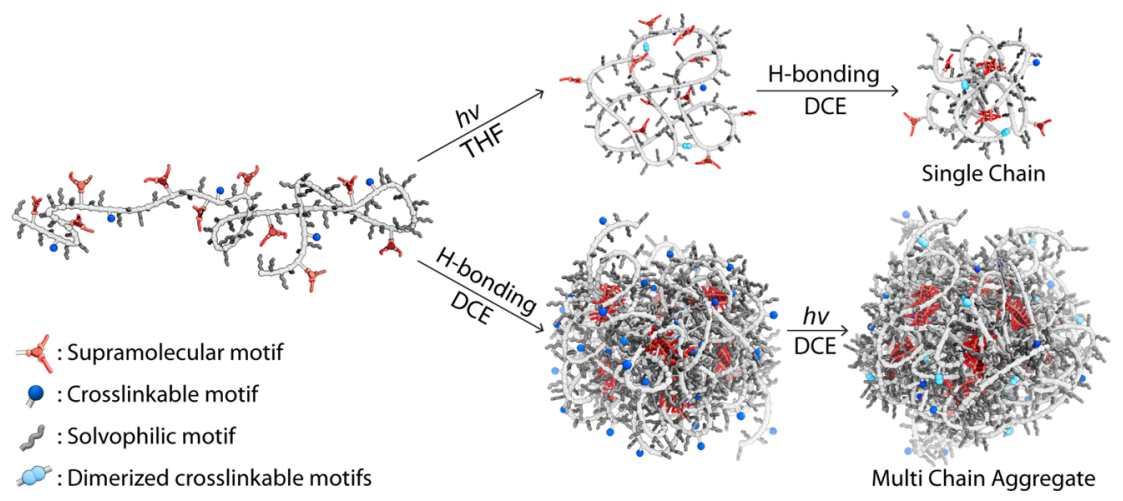

Figure 1. Schematic representation of the two pathways applied to fold and cross-link the polymers. In the first pathway (top) the coumarin pendants are first dimerized with UV-A light in THF (a solvent which prevents H bonding), followed by redissolving the particles in DCE, a solvent that promotes $\mathrm{H}$ bonding. In the second pathway (bottom), the BTA pendants are aggregated in DCE, after which the coumarin pendants are dimerized with UV-A light.

Scheme 1. Synthesis of Monomers 5 and 9 and Their Subsequent Random Copolymerization with Monomer 11 Using Reversible Addition-Fragmentation Chain-Transfer Polymerization

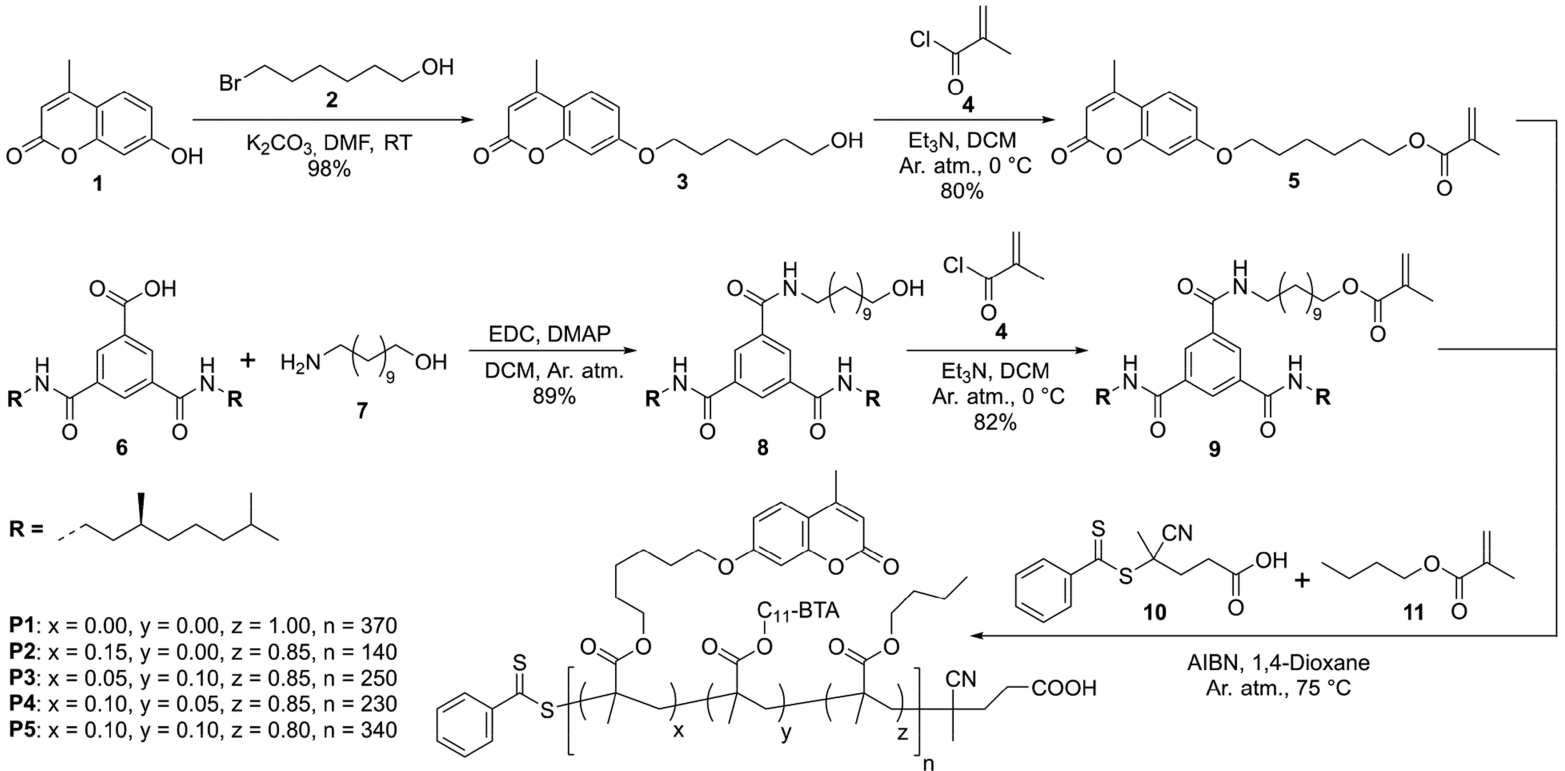

ing the protein from adopting its native fold upon subsequent removal of the urea. As a result, catalytic activity is lost.

In the past decade, numerous hydrogen-bonding supramolecular moieties have been employed to fold single polymer chains into well-defined, internally structured single-chain polymeric nanoparticles (SCPNs). Examples have been presented that apply thymine-diaminopyridine and cyanuric acid-Hamiltonian wedge pairs as well as ureidopyrimidinones, ureas, and 2-ureido-5-deazapterinines. ${ }^{13,19,34-36,22,27-33}$ In our group, the ureidopyrimidinone unit and the benzene-1,3,5tricarboxamide (BTA) moiety have been thoroughly evaluated as structuring supramolecular grafts. ${ }^{15,37,38}$ The inherent ability of BTAs to self-assemble into helical supramolecular polymers-stabilized by 3-fold hydrogen bonding-makes them suitable to provide internal structure and control the conformational flexibility of a single polymer chain in solution. ${ }^{37,39}$ In addition, different routes have been developed to control the conformation of single polymer chains via covalent cross-linking approaches, such as click chemistry, radical coupling, dimerization, and cyclization reactions. All these approaches have resulted in stably cross-linked nano- particles. ${ }^{40,41,50-52,42-49}$ Inspiring examples have been reported by the groups of Zhao, ${ }^{53,54}$ Berda, ${ }^{55}$ and Barner-Kowollik, ${ }^{56}$ in which the photodimerization of coumarin, anthracene, or pyrene derivatives was used to introduce covalent cross-links. Not only is the cross-link formation amenable to relatively mild and noninvasive conditions, it is also easy to monitor their formation using ultraviolet-visible (UV-vis) absorption spectroscopy. Furthermore, the coumarin and anthracene cross-links are in principle reversible, just like the disulfide bridges found in proteins.

In this work, we study the effect of structural constraints, induced by covalent cross-links, on the folding of a synthetic polymer chain via hydrogen-bonding interactions. Hereto, methacrylate-based monomers comprising either BTA-based or coumarin-based pendants were copolymerized with $n$-butyl methacrylate in various ratios via reversible additionfragmentation chain-transfer (RAFT) polymerization. Two folding pathways are investigated (Figure 1). In the first pathway, we covalently cross-link the coumarin pendants within the polymers in a solvent that prevents hydrogen bonding, after which folding of the polymers is induced by 


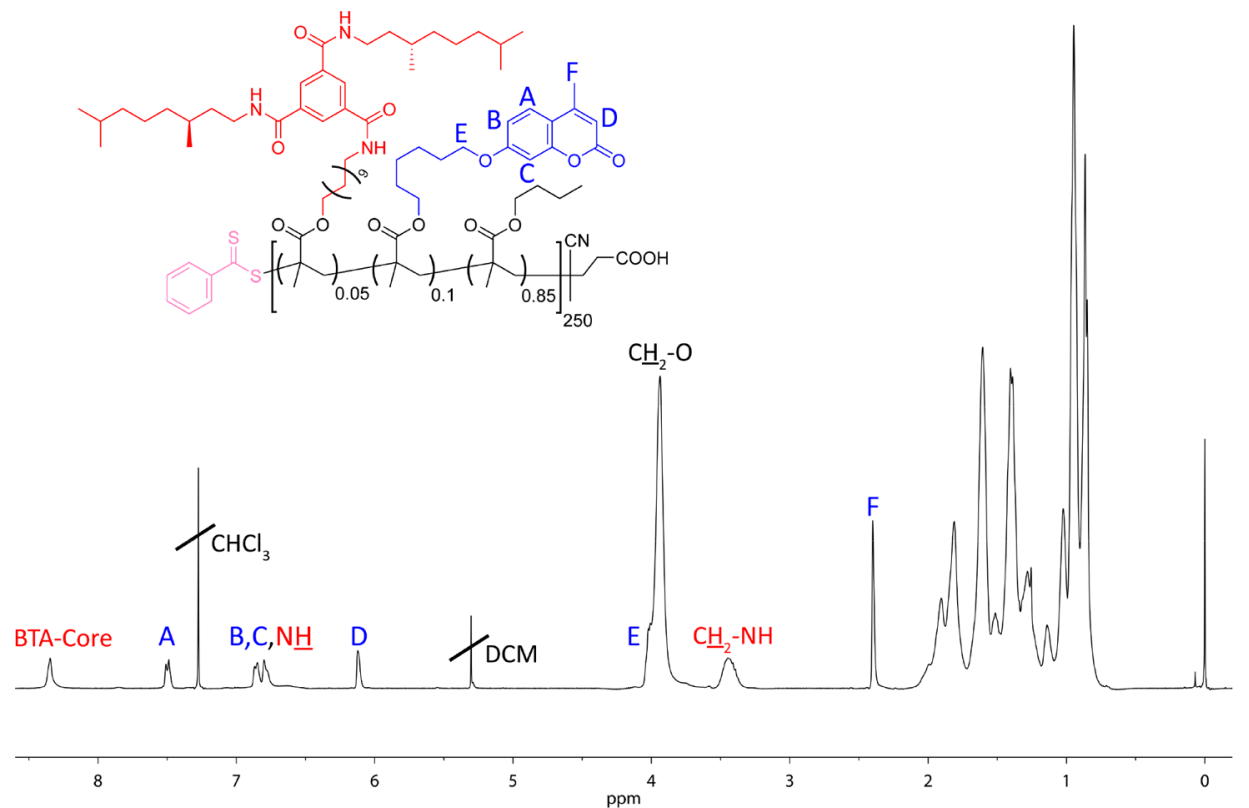

Figure 2. ${ }^{1} \mathrm{H}$ NMR spectrum of $\mathbf{P} 3$ in $\mathrm{CDCl}_{3}$.

Table 1. Overview of Polymer Composition, Degree of Polymerization (DP), Number-Average Molecular Weight $\left(M_{n}\right)$, and Dispersity $(\boxplus)$

\begin{tabular}{|c|c|c|c|c|c|c|c|}
\hline & $\mathrm{BTA}^{a}(\operatorname{mol} \%)$ & $\operatorname{coumarin}^{a}(\mathrm{~mol} \%)$ & $n$-butyl $^{a}(\operatorname{mol} \%)$ & conversion $^{b}(\%)$ & $\mathrm{DP}^{c}$ & $M_{\mathrm{n}, \mathrm{SEC}}{ }^{d}\left(\mathrm{~kg} \mathrm{~mol}^{-1}\right)$ & $\bigoplus^{d}$ \\
\hline P1 & 0 & 0 & 100 & 88 & 370 & 52.5 & 1.05 \\
\hline P2 & 0 & 15 & 85 & 68 & 140 & 24.7 & 1.13 \\
\hline P3 & 5 & 10 & 85 & 85 & 250 & 30.2 & 1.12 \\
\hline P4 & 10 & 5 & 85 & 80 & 230 & 40.8 & 1.29 \\
\hline P5 & 10 & 10 & 80 & 85 & 340 & 75.1 & 1.17 \\
\hline
\end{tabular}

${ }^{a}$ Based on the feed ratio and confirmed using ${ }^{1} \mathrm{H}$ NMR spectroscopy. ${ }^{b}$ Determined using ${ }^{1} \mathrm{H}$ NMR spectroscopy. ${ }^{c}$ Based on the conversion as determined using ${ }^{1} \mathrm{H}$ NMR spectroscopy, ${ }^{d}$ Determined using SEC in THF, calibrated with poly(styrene).

hydrogen bonding. In the second pathway, we induce hydrogen-bonding interactions between tethered BTAs prior to covalent cross-linking of the coumarin pendants. A combination of circular dichroism (CD) spectroscopy, UVvis spectroscopy, size-exclusion chromatography (SEC), and dynamic light scattering (DLS) is employed to understand the effect of the structural constraints on the folding behavior of these synthetic polymers. To our surprise, the two systems show a fundamentally different response to changes in temperature, indicating that also in synthetic polymers differences in folding pathway induce differences in the properties of the resultant nanostructures.

\section{RESULTS}

Synthesis and Characterization of the Monomers and (Co)polymers. To obtain foldable copolymers containing photo-cross-linkable groups, methacrylate monomers containing either a 4-methylcoumarin group or a chiral BTA supramolecular motif were synthesized (Scheme 1). Hereto, 4-methylcoumarin (1) was coupled to 6-bromo-1-hexanol (2) via a Williamson ether synthesis. Subsequent reaction of this product (3) with methacryloyl chloride (4) resulted in the 4methylcoumarin-functionalized methacrylate monomer $(5){ }^{54}$ Similarly, a BTA-functionalized methacrylate monomer was synthesized by extending a desymmetrized chiral BTA core (6) with an 11-amino-1-undecanol linker (7) using an $\mathrm{N}$-(3(dimethylamino)propyl)- $N^{\prime}$-ethylcarbodiimide hydrochloride
(EDC) coupling. ${ }^{36}$ The remaining alcohol group (8) was reacted with methacryloyl chloride (4) to obtain the BTAcontaining monomer (9). ${ }^{39}$ All compounds were fully characterized using ${ }^{1} \mathrm{H}$ NMR spectroscopy, ${ }^{13} \mathrm{C}$ NMR spectroscopy, FT-IR spectroscopy, and MALDI-TOF MS (Figures $\mathrm{S} 1-\mathrm{S} 8$ ).

Monomers 5 and 9 were copolymerized with $n$-butyl methacrylate (11) in various ratios using reversible addition-fragmentation chain-transfer (RAFT) polymerization. The degree of polymerization (DP) was estimated from the chain transfer agent $(\mathbf{1 0})$ to monomer ratio in combination with the final monomer conversion as determined by ${ }^{1} \mathrm{H}$ NMR spectroscopy. After purification of the polymers, via precipitation in cold methanol, the composition of the polymers was determined using ${ }^{1} \mathrm{H}$ NMR spectroscopy (see Figure 2 for P3 and Figures S9-S13 for P1-P5). The BTA incorporation was based on the ratio between the peak corresponding to the methylene protons next to the amides $(\delta 3.57-3.31 \mathrm{ppm}, 6 \mathrm{H})$ and the peak associated with the methylene protons next to the ester groups in the polymer backbone $(\delta 4.17-3.66 \mathrm{ppm}, 6 \mathrm{H})$. Similarly, the 4-methylcoumarin incorporation was estimated by comparing the signal corresponding to the methyl group in 4-methylcoumarin $(\delta 2.40,3 \mathrm{H})$ with the methylene protons next to the ester groups in the polymer backbone $(\delta 4.17-3.66$ $\mathrm{ppm}, 6 \mathrm{H})$. The values obtained for the incorporation of both the BTA and the 4-methylcoumarin monomer matched well with the feed ratios (Table 1). 
Scheme 2. Covalent Cross-Linking of P2 via UV-A-Induced 4-Methylcoumarin Dimerization
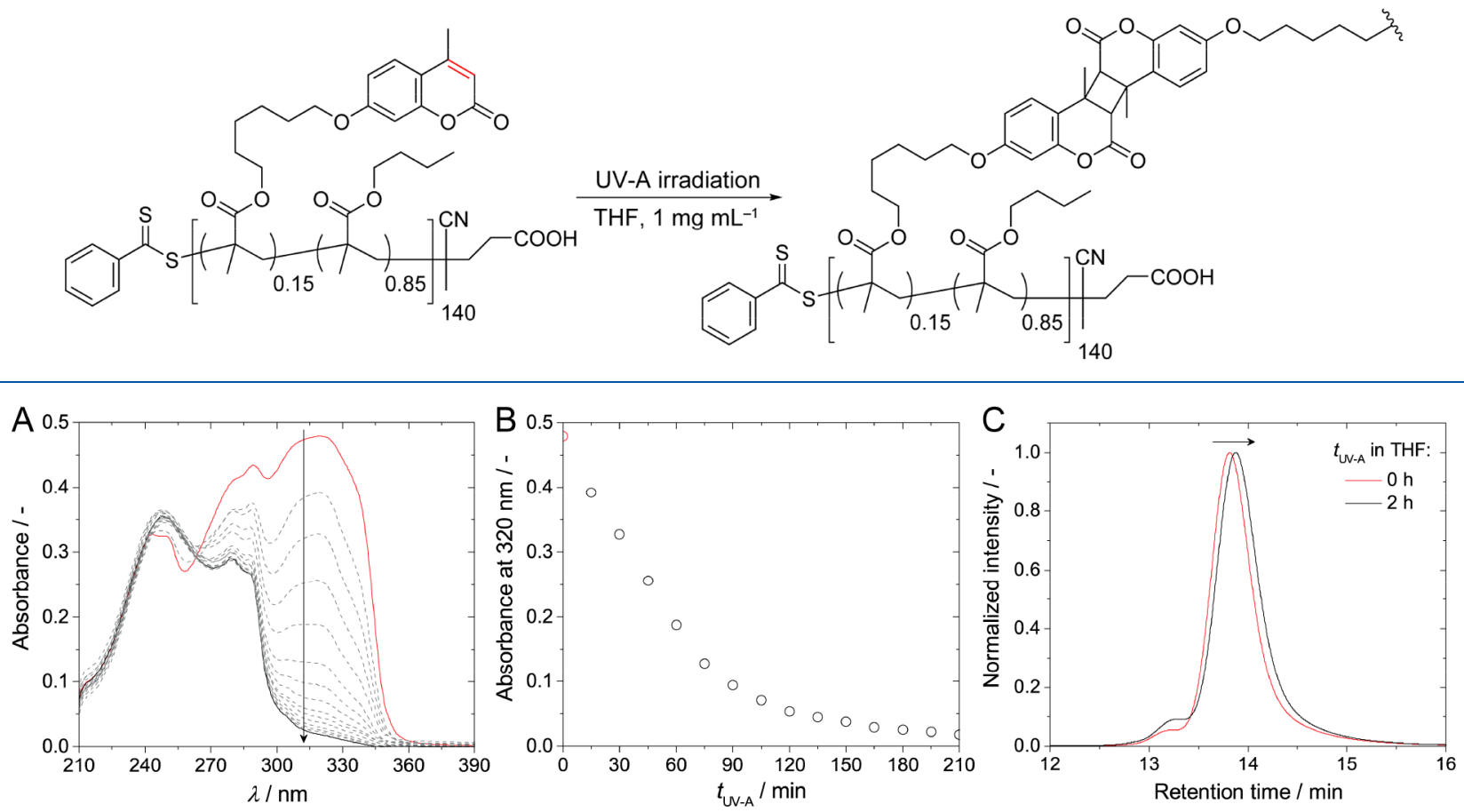

Figure 3. (A) UV-vis spectra of $\mathbf{P} 2$ in THF as a function of the UV-A irradiation $\left(c_{\text {polymer }}=1.0 \mathrm{mg} \mathrm{mL}^{-1}, l=0.2 \mathrm{~cm}\right)$. (B) Absorbance at $320 \mathrm{~nm}$ as a function of the UV-A exposure time. (C) SEC trace of P2 before and after $2 \mathrm{~h}$ of irradiation with UV-A light in THF.

Covalent Cross-Linking of Polymers via UV-A Induced 4-Methylcoumarin Dimerization. Coumarin and its derivatives photodimerize upon irradiation with ultraviolet A (UV-A) light $(\lambda=310-400 \mathrm{~nm})$, also when they are attached to a polymer backbone. ${ }^{53}$ This photodimerization process proceeds via a $[2+2]$ photocycloaddition in which the double bonds of two coumarin moieties are converted into a single connecting cyclobutane ring. ${ }^{57}$ To illustrate this process, we discuss the photodimerization of $\mathbf{P 2}$ (Scheme 2) in more detail.

UV-vis absorption spectroscopy was used to monitor the dimerization of the coumarin derivatives by probing in time the change in the conjugated system upon conversion of the double bonds into single bonds. To promote the intramolecular covalent cross-linking of the polymers in solution, aggregation must be suppressed. Hereto, the covalent crosslinking is preferably performed in a good solvent at a low concentration. In the case of reference polymer P2, dynamic light scattering (DLS) measurements show that the polymer in THF has a hydrodynamic radius $\left(R_{\mathrm{H}}\right)$ of $3.8 \mathrm{~nm}$ (Figure $\mathrm{S} 14$ ). This shows that the copolymers are highly soluble in THF, most likely showing single-chain character up to concentrations of at least $10 \mathrm{mg} \mathrm{mL}{ }^{-1} .^{58}$ Upon the prolonged irradiation with UV-A light $\left(11.5 \mathrm{~mW} \mathrm{~cm}^{-2}\right)$, the 4methylcoumarin moieties start to dimerize as evidenced by the decreasing band in the 300-360 nm regime of the UV-vis absorption spectra (Figure 3A, Figures S15 and S19 for P4). The monotonous decay of this band during $210 \mathrm{~min}$ of UV-A irradiation demonstrates the gradual nature of the covalent cross-linking process (Figure 3B).

If the covalent cross-linking occurs predominantly intramolecularly, the size of the cross-linked polymer is expected to become smaller. This reduction in size is observed using sizeexclusion chromatography, which probes the hydrodynamic diameter of the polymers in solution. The SEC trace of P2 shifts to longer retention times after exposure to UV-A light (Figure 3C), indicating a reduction in the hydrodynamic volume of the polymer coil. Importantly, the dispersity $(\boxplus)$ of the polymer increases only slightly as a result of the crosslinking step (from $Ð=1.12$ to 1.15 ), indicating the intramolecular nature of the cross-link formation as well as the absence of degradation processes. The SEC trace of reference polymer P1, which does not contain coumarin units, appears to be mostly unaffected by irradiation with UV-A light (Figure S20A).

Effect of Covalent Cross-Linking on the Copolymer's Folding Behavior. The ability of the chiral BTA grafts to form helical, hydrogen-bonded assemblies with a preferred handedness can be used to fold the polymer backbone into a structured nanoparticle via supramolecular cross-linking. The presence and formation of such structured domains can be probed using circular dichroism (CD) spectroscopy. A negative $\mathrm{CD}$ effect is indicative for the formation of lefthanded helical BTA assemblies. ${ }^{59} \mathrm{CD}$ cooling curves are obtained by monitoring the CD effect at $225 \mathrm{~nm}$ as a function of temperature. The cooling curves provide information about the stability of the supramolecular cross-links and their selfassembly mechanism.

As shown in the previous section, THF is an excellent solvent to intramolecularly form covalent cross-links within the polymer chains in solution. However, THF also interferes with the hydrogen-bonding interactions, preventing the selfassembly of the BTAs. Taking P4 as an example, the polymer was first covalently cross-linked in THF and after evaporation of the solvent redissolved in 1,2-dichloroethane (DCE), a solvent that promotes hydrogen-bond formation. This sample preparation procedure resulted in a solution that shows a clear, negative CD effect (Figure S21A). This indicates that despite 

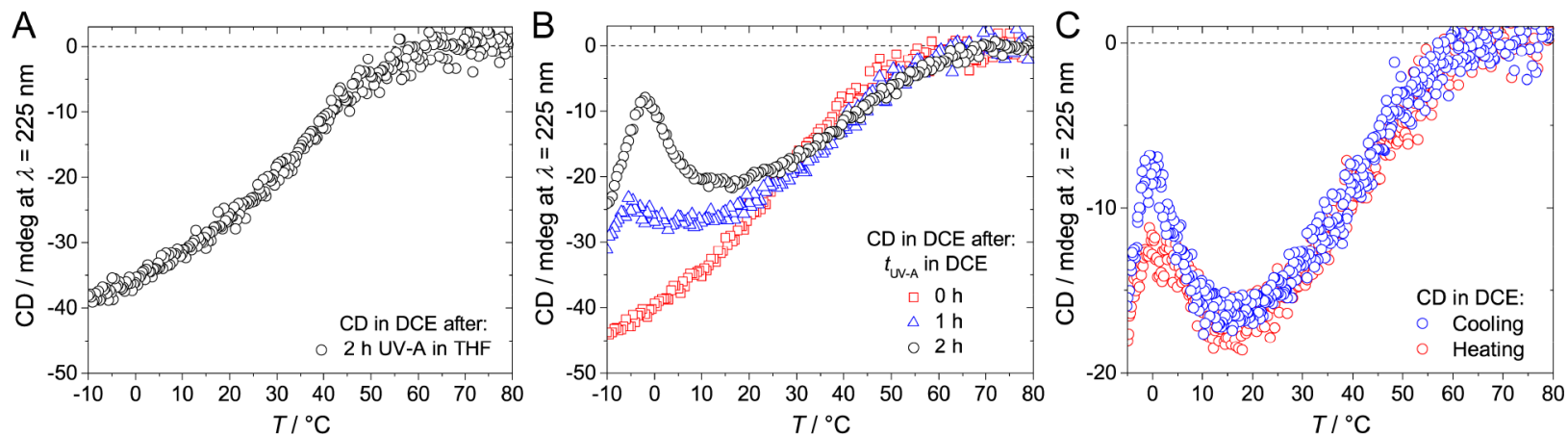

Figure 4. CD cooling curves of $\mathbf{P 4}$ in DCE $\left(c_{\text {polymer }}=0.18 \mathrm{mg} \mathrm{mL}^{-1}, \lambda=225 \mathrm{~nm}\right)$. (A) CD cooling curve of P4 cross-linked in THF and subsequently transferred to DCE. (B) CD cooling curves of $\mathbf{P 4}$ as a function of the time exposed to UV-A light in DCE. (C) CD cooling curve and the subsequent $\mathrm{CD}$ heating curve of $\mathbf{P} 4$ after $2 \mathrm{~h}$ cross-linking in DCE.
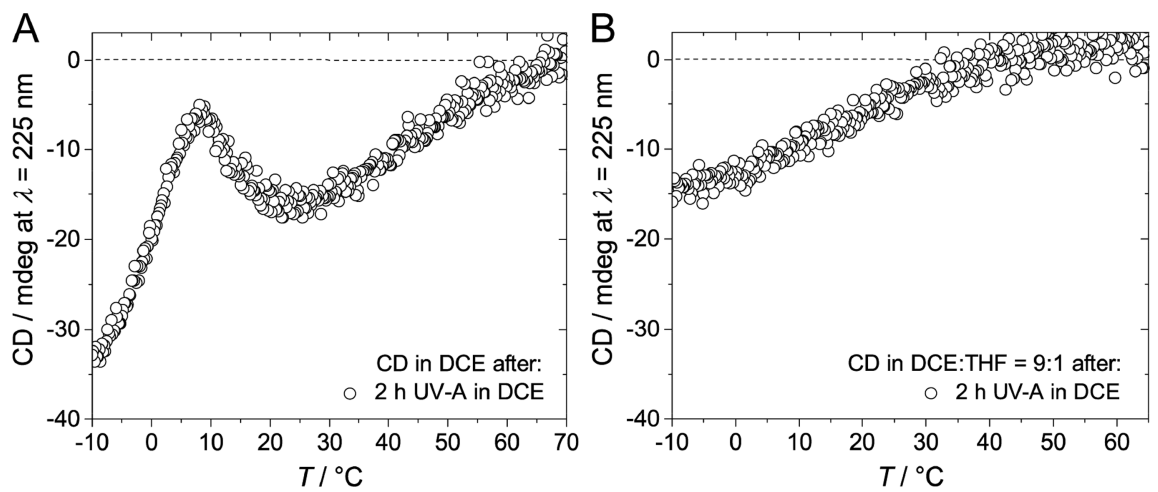

Figure 5. (A) CD cooling curve of $\mathbf{P 4}$ in DCE after $2 \mathrm{~h}$ cross-linking in DCE $\left(c_{\text {polymer }}=0.18 \mathrm{mg} \mathrm{mL}^{-1}, \lambda=225 \mathrm{~nm}, t_{\mathrm{UV}-\mathrm{A}}=2 \mathrm{~h}\right)$. (B) CD cooling curve of P4 in DCE:THF $=9: 1$ after $2 \mathrm{~h}$ cross-linking in DCE $\left(c_{\text {polymer }}=0.16 \mathrm{mg} \mathrm{mL}^{-1}, \lambda=225 \mathrm{~nm}\right)$.

the presence of covalent cross-links, the system is capable of reforming hydrogen bonds between the pendant BTAs. The CD cooling curve (Figure 4A) is similar to those previously observed upon intramolecular assembly of chiral supramolecular moieties grafted to a polymer backbone. ${ }^{2,15,18,60}$

We then investigate the behavior of P4 in DCE. Before the exposure of the polymer solution to any UV-A light, the cooling curve of P4 in DCE (Figure 4B, red line) is nearly identical to the one shown in Figure $4 \mathrm{~A}$, indicating that irrespective of the presence of covalent cross-links the systems behave the same. Remarkably, irradiation of P4 in DCE with UV-A light, at room temperature, did not significantly alter the amplitude of the sample's CD effect at room temperature, indicating that the extent to which the supramolecular assemblies are formed is not altered by the formation of the covalent cross-links (Figure S22). However, irradiation of the polymer in DCE resulted in a distinct change in the shape of the cooling curve below $T=20{ }^{\circ} \mathrm{C}$. The nature of the curve became more and more nonmonotonic upon the prolonged irradiation with UV-A light, as indicated by the distinct feature observed in the low temperature regime of the cooling curves (Figure 4B, blue and black curves). Repeating the experiment multiple times showed that the transition from the commonly observed to the nonmonotonic cooling curve was reproducible. However, the exact magnitude and temperature at which the additional feature is occurring vary from sample to sample (Figures 4B,C and 5A, Figures S23 and S24). Furthermore, time-dependent measurements, probing the $\mathrm{CD}$ effect at the temperature of the maximum of the nonmonotonic feature, showed that the signal is stable over time (Figure S23). Moreover, heating and cooling experiments showed that the process is reversible as a function of temperature (Figure 4C). Polymer P5, with a higher DP and coumarin content, also exhibits the nonmonotonic behavior in the $\mathrm{CD}$ cooling curves (Figure S25).

Similar, nonmonotonic cooling curves have been observed in prior research focusing on the self-assembly of "free" BTAs in alkane solvents. There, on the basis of changes in the shape of the CD spectra, it was concluded that the conformation of the hydrogen-bonded amides of the BTAs in the self-assembled state changed as a function of temperature. ${ }^{61}$ Accordingly, the full $\mathrm{CD}$ spectra were acquired at various points along a nonmonotonic cooling curve. While the magnitude of the CD signals varied with temperature, the shape of the spectra remained the same over the entire temperature regime (Figure S24). This indicates that the nonmonotonic nature of the cooling curve is proposedly not related to changes in the conformation of the hydrogen-bonded amides, but rather to a polymerization-depolymerization mechanism and/or a change in the $P$ over $M$ ratio of the stacks.

Interestingly, adding $10 \mathrm{vol} \%$ of THF to a sample exhibiting the nonmonotonic behavior causes the cooling curve to adopt an isodesmic shape (Figure 5B). Besides the change in the shape of the cooling curve, also the magnitude of the CD effect decreases significantly. This is attributed to THF's ability to interfere with the stabilizing hydrogen-bonding interactions of the BTA stacks, thereby reducing the supramolecular graft's tendency to self-assemble.

Effect of Temperature and UV-A Irradiation on the Copolymer's Solubility. To acquire more insight into the remarkable cooling curves of P4 and P5 after cross-linking in DCE, the behavior of the copolymers in solution was studied 

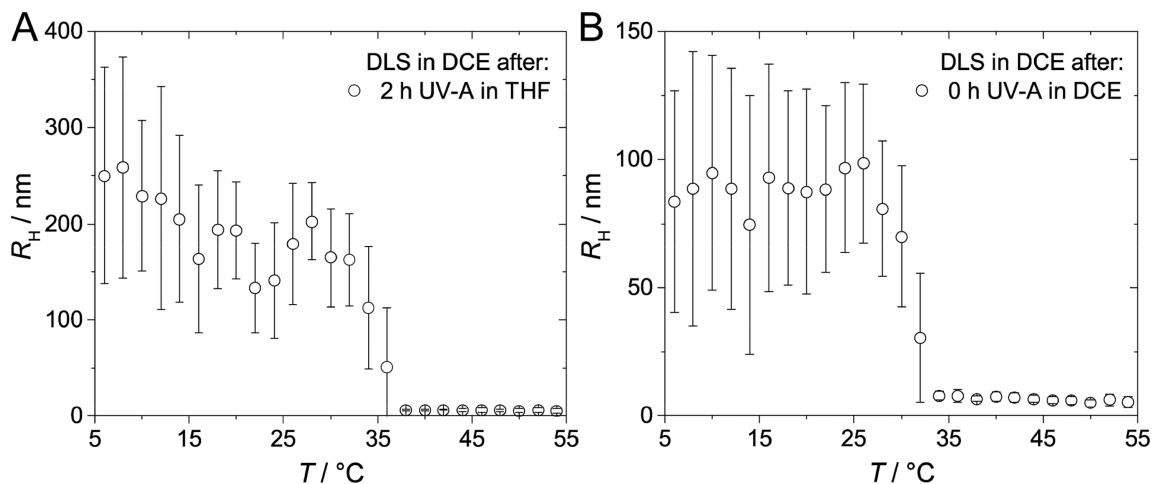

Figure 6. Hydrodynamic radius of P5 in DCE $\left(c_{\text {polymer }}=0.1 \mathrm{mg} \mathrm{mL}^{-1}\right)$ as a function of temperature: $(\mathrm{A})$ after exposure to $2 \mathrm{~h}$ of UV-A irradiation in THF and (B) before any exposure to UV-A light.

further by dynamic light scattering (DLS) experiments. Here we focus on P5 because its higher DP results in slightly larger nanoparticles, allowing a more accurate analysis using DLS, but P4 shows similar results. The hydrodynamic radius $\left(R_{\mathrm{H}}\right)$ of P5 was investigated as a function of temperature. In THF, P5 shows an $R_{\mathrm{H}}$ of $6 \mathrm{~nm}$ before irradiation with UV-A light (Figure S16B). These dimensions are comparable to those found for other individual polymers chains of similar chemical nature and molecular weight, indicating that the polymer is present as individual chains in THF. ${ }^{36,62}$ Consequently, upon the irradiation with UV-A light, the particles are covalently cross-linked as individual polymers in solution, and the size of the particles decreases (Figure S16B). Upon subsequent evaporation of all of the THF and redissolution of the intramolecularly cross-linked particles in DCE, a clear temperature-dependent particle size is observed in DLS (Figure 6A). At high temperatures, the polymers are present as small particles $\left(R_{\mathrm{H}}=5.6 \pm 0.9 \mathrm{~nm}\right)$. Upon cooling, the particles start to aggregate into large multichain aggregates. The temperature-dependent behavior of P5 in DCE, before any irradiation with UV-A light, reveals a similar temperature dependence. At high temperatures the polymer solution consists of small particles $\left(R_{\mathrm{H}}=9.2 \pm 1.4 \mathrm{~nm}\right)$ which start to cluster into large aggregates as soon as the temperature drops below $34{ }^{\circ} \mathrm{C}$ (Figure 6B). To investigate to which extent this behavior is driven by BTA-BTA interactions, we also evaluated the temperature-dependent DLS of reference polymer P1 that lacks pendant BTA units. Also in this case, an upper critical solution temperature (UCST) behavior is observed, indicating that the propensity to aggregate is an intrinsic property of the poly( $n$-butyl methacrylate) backbone in DCE (Figure S18).

Based on the observed temperature-dependent behavior of P4 and P5 in DCE, performing the covalent cross-linking step at room temperature in DCE should result in the formation of intermolecularly covalently cross-linked aggregates. If this is the case, the polymer aggregates are no longer able to dissociate into individual polymer chains at elevated temperatures. As a result of this lack in dynamics, the covalently crosslinked polymers are expected to remain large over the entire temperature range. However, experimental verification by DLS at low concentration failed due to contamination with dust, which could not be removed via filtration without the simultaneous loss of the covalently cross-linked polymer aggregates. When irradiating a polymer solution at a 10 -fold higher concentration in DCE $\left(c_{\text {polymer }}=1.0 \mathrm{mg} \mathrm{mL}^{-1}\right)$, aggregates were visible to the naked eye (Figure S27A,B).
These aggregates remained visible up to $70{ }^{\circ} \mathrm{C}$. By contrast, when cross-linked in THF and subsequently transferred to DCE, no such aggregates were observed at elevated temperatures.

\section{DISCUSSION}

In THF, BTA-pendant polymers are present as individual polymer chains in solution $\left(R_{\mathrm{H}}=6 \mathrm{~nm}\right)$. As a result, exposure to UV-A light results in intramolecular covalent cross-linking of the polymers. When exchanging the solvent from THF to DCE, these covalently cross-linked polymers exhibit an interesting temperature-dependent behavior: upon decreasing the temperature, the magnitude of the CD effect gradually increases, but the size of the particles suddenly increases. From DLS in combination with CD measurements we observe that above $70{ }^{\circ} \mathrm{C}$ the polymers are present as individual nanoparticles in DCE $\left(R_{\mathrm{H}}\right.$ around $\left.5.6 \mathrm{~nm}\right)$ with no supramolecular interactions between the BTAs. Upon cooling the solution, the pendant BTAs start to self-assemble. Initially the magnitude of the $\mathrm{CD}$ effect increases, but the particles remain small. Below $40{ }^{\circ} \mathrm{C}$, the sudden formation of large, multichain aggregates with $R_{\mathrm{H}}>50 \mathrm{~nm}$ is observed (Figure 7A). The reversible nature of this transition suggests that the

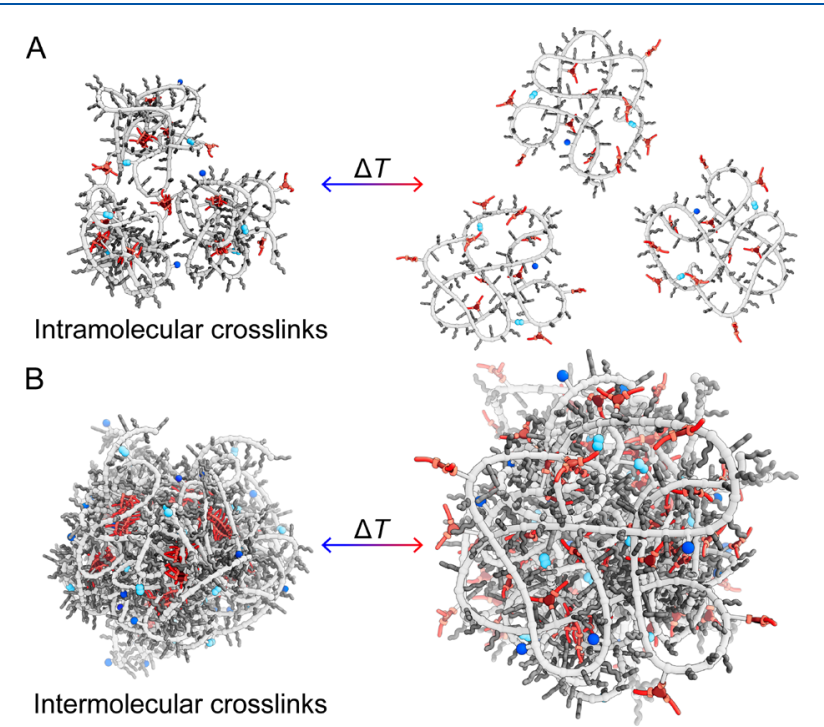

Figure 7. Schematic representation of the UCST-driven behavior of the polymers in DCE, in case the covalent cross-links are formed (A) intramolecularly and (B) intermolecularly. 
formed aggregates do not entangle significantly at those time scales and therefore remain dynamic. By comparing the temperature dependence observed in DLS with the cooling curve obtained using $\mathrm{CD}$ spectroscopy, we conclude that the $\mathrm{CD}$ effect upon cooling from 80 to $38^{\circ} \mathrm{C}$ can be attributed to the self-assembly of BTAs present in a single polymer particle. Upon further cooling of the solution, the individual polymeric nanoparticles start to cluster into large aggregates, a result of the UCST of the polymethacrylate backbone in DCE. Therefore, the $\mathrm{CD}$ effect below $38{ }^{\circ} \mathrm{C}$ is attributable to both intra- and interpolymer BTA self-assembly. Interestingly, at this transition temperature the slope of the $\mathrm{CD}$ cooling curve seems to become less steep. Presumably, the mobility of the BTAs is slightly more restricted in the aggregated state compared to the single-chain state.

In DCE, in contrast, BTA-pendant polymers are present as small particles $\left(R_{\mathrm{H}}=9.2 \pm 1.4 \mathrm{~nm}\right)$ at higher temperatures, but a sudden and strong increase of the particle size occurs below $34{ }^{\circ} \mathrm{C}$ into multichain aggregates with $R_{\mathrm{H}}>75 \mathrm{~nm}$ at room temperature. The origin of the sudden increase in size is attributed to the UCST of the polymethacrylate backbone in DCE. Also in this case, the magnitude of the CD effect gradually increases upon decreasing the temperature. As a result, irradiating the solution at room temperature results in a covalently cross-linked polymer network in which BTAs are still capable of aggregation, but the network affects the degree to which this is possible (Figure 7B). The self-assembly of the BTAs in this constrained state is characterized by intriguing nonmonotonic behavior in $\mathrm{CD}$ spectroscopy. Above $20^{\circ} \mathrm{C}$, the BTAs can self-assemble in a normal, rather unhindered, way. However, below this temperature the network contracts, first further hampering the BTA self-assembly and later even disrupting the formed BTA stacks. Interestingly, the nonmonotonic shape of the cooling curves suggests that further cooling of the solution results in the repolymerization of BTAs in the network. We speculate that this behavior is observed because the compactness of the network remains constant in the low-temperature regime while the tendency of the BTAs to self-assemble increases with decreasing temperature. This hypothesis is supported by the observation that the addition of $10 \mathrm{vol} \%$ of THF, a good solvent swelling the cross-linked aggregates, transforms a nonmonotonic cooling curve into a typical isodesmic one. Presumably, swelling of the network reduces the constraints imposed by the cross-links, providing the BTAs with the space needed to exhibit their typical isodesmic self-assembly behavior.

\section{CONCLUSIONS}

The effect of structural constraints, induced by covalent crosslinks, on the folding of a synthetic polymer chain via hydrogenbonding interactions was investigated. Hereto, foldable copolymers consisting of BTA- and coumarin-based pendants on a poly ( $n$-butyl methacrylate) backbone were studied using a combination of CD spectroscopy, UV-vis spectroscopy, SEC, and DLS. The results show that in synthetic foldable polymers the order of events matters greatly and determines the outcome of the formation of folded polymers. By use of a solvent in which hydrogen bonding is in effect prevents the formation of SCPNs, the subsequent covalent cross-linking of the system results in fixated multichain aggregates. By contrast, covalently cross-linking the system when no hydrogen bonds are present favors the formation of SCPNs. Subsequent initiation of the hydrogen-bonding interactions by cooling of the solution results in the intramolecular self-assembly of the pendant BTAs. However, at a specific temperature the individual nanoparticles cluster into large multichain aggregates, also allowing intermolecular BTA self-assembly. The intra- and intermolecularly cross-linked systems show a fundamentally different response to changes in temperature. While the intramolecularly cross-linked systems remain dynamic, the self-assembly dynamics of the BTAs in the cross-linked multichain aggregates is characterized by intriguing nonmonotonic behavior in CD spectroscopy. This indicates that the order of events controls the pathways leading to different properties in the formed nanostructures.

This research further elucidates the interplay between the structural design of foldable polymers and their behavior in solution. Not only does it provide more insight into the effect of structural constraints on the folding behavior and mechanism, it also highlights the importance of studying the system's response to changes in temperature in more detail. Furthermore, it offers new understanding in the limits of the current analytical techniques used to study such supramolecular systems.

\section{ASSOCIATED CONTENT}

\section{S Supporting Information}

The Supporting Information is available free of charge on the ACS Publications website at DOI: 10.1021/acs.macromol.8b01623.

Detailed synthetic and experimental procedures, ${ }^{1} \mathrm{H}$ NMR spectroscopy, dynamic light scattering, circular dichroism spectroscopy, size-exclusion chromatography, and UV-vis absorption spectroscopy (PDF)

\section{AUTHOR INFORMATION}

\section{Corresponding Authors}

*(A.R.A.P.) E-mail a.palmans@tue.nl, tel 0031402473105.

*(I.K.V.) E-mail i.voets@tue.nl, tel 0031402475303.

*(E.W.M.) E-mail e.w.meijer@tue.nl, tel 0031402473101. ORCID

Ilja K. Voets: 0000-0003-3543-4821

Anja R. A. Palmans: 0000-0002-7201-1548

E. W. Meijer: 0000-0003-4126-7492

Notes

The authors declare no competing financial interest.

\section{ACKNOWLEDGMENTS}

This work was financed by the Dutch Ministry of Education, Culture and Science (Gravity program 024.001.035). I.K.V. is grateful for financial support from The Netherlands Organization for Scientific research (NWO VIDI grant: 723.014.006, ECHO-STIP 717.013.005). The ICMS Animation Studio (Eindhoven University of Technology) is acknowledged for providing the artwork.

\section{REFERENCES}

(1) Yang, L.; Tan, X.; Wang, Z.; Zhang, X. Supramolecular Polymers: Historical Development, Preparation, Characterization, and Functions. Chem. Rev. 2015, 115 (15), 7196-7239.

(2) De Greef, T. F. A.; Smulders, M. M. J.; Wolffs, M.; Schenning, A. P. H. J.; Sijbesma, R. P.; Meijer, E. W. Supramolecular Polymerization. Chem. Rev. 2009, 109 (11), 5687-5754.

(3) Yashima, E.; Ousaka, N.; Taura, D.; Shimomura, K.; Ikai, T.; Maeda, K. Supramolecular Helical Systems: Helical Assemblies of 
Small Molecules, Foldamers, and Polymers with Chiral Amplification and Their Functions. Chem. Rev. 2016, 116 (22), 13752-13990.

(4) Krieg, E.; Bastings, M. M. C.; Besenius, P.; Rybtchinski, B. Supramolecular Polymers in Aqueous Media. Chem. Rev. 2016, 116 (4), 2414-2477.

(5) Huang, Z.; Qin, B.; Chen, L.; Xu, J. F.; Faul, C. F. J.; Zhang, X. Supramolecular Polymerization from Controllable Fabrication to Living Polymerization. Macromol. Rapid Commun. 2017, 38 (17), 1700312.

(6) Sorrenti, A.; Leira-Iglesias, J.; Markvoort, A. J.; de Greef, T. F. A.; Hermans, T. M. Non-Equilibrium Supramolecular Polymerization. Chem. Soc. Rev. 2017, 46 (18), 5476-5490.

(7) Besenius, P. Controlling Supramolecular Polymerization through Multicomponent Self-Assembly. J. Polym. Sci., Part A: Polym. Chem. 2017, 55 (1), 34-78.

(8) Mavila, S.; Eivgi, O.; Berkovich, I.; Lemcoff, N. G. Intramolecular Cross-Linking Methodologies for the Synthesis of Polymer Nanoparticles. Chem. Rev. 2016, 116 (3), 878-961.

(9) Lyon, C. K.; Prasher, A.; Hanlon, A. M.; Tuten, B. T.; Tooley, C. A.; Frank, P. G.; Berda, E. B. A Brief User's Guide to Single-Chain Nanoparticles. Polym. Chem. 2015, 6 (2), 181-197.

(10) Huo, M.; Wang, N.; Fang, T.; Sun, M.; Wei, Y.; Yuan, J. SingleChain Polymer Nanoparticles: Mimic the Proteins. Polymer 2015, 66, A11-A21.

(11) Deans, R.; Ilhan, F.; Rotello, V. M. Recognition-Mediated Unfolding of a Self-Assembled Polymeric Globule. Macromolecules 1999, 32 (15), 4956-4960.

(12) Wu, C.; Wang, X. Globule-to-Coil Transition of a Single Homopolymer Chain in Solution. Phys. Rev. Lett. 1998, 80 (18), 4092-4094.

(13) Seo, M.; Beck, B. J.; Paulusse, J. M. J.; Hawker, C. J.; Kim, S. Y. Polymeric Nanoparticles via Noncovalent Cross-Linking of Linear Chains. Macromolecules 2008, 41 (17), 6413-6418.

(14) Appel, E. A.; Dyson, J.; del Barrio, J.; Walsh, Z.; Scherman, O. A. Formation of Single-Chain Polymer Nanoparticles in Water through Host-Guest Interactions. Angew. Chem., Int. Ed. 2012, 51 (17), 4185-4189.

(15) Hosono, N.; Gillissen, M. A. J.; Li, Y.; Sheiko, S. S.; Palmans, A. R. A.; Meijer, E. W. Orthogonal Self-Assembly in Folding Block Copolymers. J. Am. Chem. Soc. 2013, 135 (1), 501-510.

(16) Lu, J.; Ten Brummelhuis, N.; Weck, M. Intramolecular Folding of Triblock Copolymers via Quadrupole Interactions between Poly(Styrene) and Poly(Pentafluorostyrene) Blocks. Chem. Commun. 2014, 50 (47), 6225-6227.

(17) Knöfel, N. D.; Rothfuss, H.; Willenbacher, J.; Barner-Kowollik, C.; Roesky, P. W. Platinum(II)-Crosslinked Single-Chain Nanoparticles: An Approach towards Recyclable Homogeneous Catalysts. Angew. Chem., Int. Ed. 2017, 56 (18), 4950-4954.

(18) Altintas, O.; Artar, M.; Ter Huurne, G.; Voets, I. K.; Palmans, A. R. A.; Barner-Kowollik, C.; Meijer, E. W. Design and Synthesis of Triblock Copolymers for Creating Complex Secondary Structures by Orthogonal Self-Assembly. Macromolecules 2015, 48 (24), 89218932.

(19) Cheng, C.-C.; Chang, F.-C.; Yen, H.-C.; Lee, D.-J.; Chiu, C.W.; Xin, Z. Supramolecular Assembly Mediates the Formation of Single-Chain Polymeric Nanoparticles. ACS Macro Lett. 2015, 4 (10), $1184-1188$.

(20) Wang, F.; Pu, H.; Jin, M.; Wan, D. Supramolecular Nanoparticles via Single-Chain Folding Driven by Ferrous Ions. Macromol. Rapid Commun. 2016, 37 (4), 330-336.

(21) Wang, F.; Pu, H.; Che, X. Voltage-Responsive Single-Chain Polymer Nanoparticles via Host-guest Interaction. Chem. Commun. 2016, 52 (17), 3516-3519.

(22) Matsumoto, K.; Terashima, T.; Sugita, T.; Takenaka, M.; Sawamoto, M. Amphiphilic Random Copolymers with Hydrophobic/ Hydrogen-Bonding Urea Pendants: Self-Folding Polymers in Aqueous and Organic Media. Macromolecules 2016, 49 (20), 7917-7927.

(23) Fischer, T. S.; Schulze-Sünninghausen, D.; Luy, B.; Altintas, O.; Barner-Kowollik, C. Stepwise Unfolding of Single-Chain Nano- particles by Chemically Triggered Gates. Angew. Chem., Int. Ed. 2016, 55 (37), 11276-11280.

(24) Ogura, Y.; Artar, M.; Palmans, A. R. A.; Sawamoto, M.; Meijer, E. W.; Terashima, T. Self-Assembly of Hydrogen-Bonding Gradient Copolymers: Sequence Control via Tandem Living Radical Polymerization with Transesterification. Macromolecules 2017, 50 (8), 32153223.

(25) Ślęczkowski, M. L.; Meijer, E. W.; Palmans, A. R. A. Cooperative Folding of Linear Poly(Dimethyl Siloxane)s via Supramolecular Interactions. Macromol. Rapid Commun. 2017, 38 (24), 1700566.

(26) Anfinsen, C. B. Principles That Govern the Folding of Protein Chains. Science 1973, 181 (4096), 223-230.

(27) Foster, E. J.; Berda, E. B.; Meijer, E. W. Metastable Supramolecular Polymer Nanoparticles via Intramolecular Collapse of Single Polymer Chains. J. Am. Chem. Soc. 2009, 131 (20), 69646966.

(28) Berda, E. B.; Foster, E. J.; Meijer, E. W. Toward Controlling Folding in Synthetic Polymers: Fabricating and Characterizing Supramolecular Single-Chain Nanoparticles. Macromolecules 2010, 43 (3), 1430-1437.

(29) Altintas, O.; Rudolph, T.; Barner-Kowollik, C. Single Chain Self-Assembly of Well-Defined Heterotelechelic Polymers Generated by ATRP and Click Chemistry Revisited. J. Polym. Sci., Part A: Polym. Chem. 2011, 49 (12), 2566-2576.

(30) Altintas, O.; Gerstel, P.; Dingenouts, N.; Barner-Kowollik, C. Single Chain Self-Assembly: Preparation of $\alpha, \omega$-Donor-acceptor Chains via Living Radical Polymerization and Orthogonal Conjugation. Chem. Commun. 2010, 46 (34), 6291-6293.

(31) Altintas, O.; Lejeune, E.; Gerstel, P.; Barner-Kowollik, C. Bioinspired Dual Self-Folding of Single Polymer Chains via Reversible Hydrogen Bonding. Polym. Chem. 2012, 3 (3), 640-651.

(32) Romulus, J.; Weck, M. Single-Chain Polymer Self-Assembly Using Complementary Hydrogen Bonding Units. Macromol. Rapid Commun. 2013, 34 (19), 1518-1523.

(33) Stals, P. J. M.; Gillissen, M. A. J.; Nicolaÿ, R.; Palmans, A. R. A.; Meijer, E. W. The Balance between Intramolecular Hydrogen Bonding, Polymer Solubility and Rigidity in Single-Chain Polymeric Nanoparticles. Polym. Chem. 2013, 4 (8), 2584-2597.

(34) Ter Huurne, G. M.; Gillissen, M. A. J.; Palmans, A. R. A.; Voets, I. K.; Meijer, E. W. The Coil-to-Globule Transition of Single-Chain Polymeric Nanoparticles with a Chiral Internal Secondary Structure. Macromolecules 2015, 48 (12), 3949-3956.

(35) Wang, F.; Pu, H.; Jin, M.; Pan, H.; Chang, Z.; Wan, D.; Du, J. From Single-Chain Folding to Polymer Nanoparticles via Intramolecular Quadruple Hydrogen-Bonding Interaction. J. Polym. Sci., Part A: Polym. Chem. 2015, 53 (15), 1832-1840.

(36) Ter Huurne, G. M.; De Windt, L. N. J.; Liu, Y.; Meijer, E. W.; Voets, I. K.; Palmans, A. R. A. Improving the Folding of Supramolecular Copolymers by Controlling the Assembly Pathway Complexity. Macromolecules 2017, 50 (21), 8562-8569.

(37) Cantekin, S.; de Greef, T. F. A.; Palmans, A. R. A. Benzene1,3,5-Tricarboxamide: A Versatile Ordering Moiety for Supramolecular Chemistry. Chem. Soc. Rev. 2012, 41 (18), 6125-6137.

(38) Mes, T.; van der Weegen, R.; Palmans, A. R. A.; Meijer, E. W. Single-Chain Polymeric Nanoparticles by Stepwise Folding. Angew. Chem., Int. Ed. 2011, 50 (22), 5085-5089.

(39) Terashima, T.; Mes, T.; De Greef, T. F. A.; Gillissen, M. A. J.; Besenius, P.; Palmans, A. R. A.; Meijer, E. W. Single-Chain Folding of Polymers for Catalytic Systems in Water. J. Am. Chem. Soc. 2011, 133 (13), 4742-4745.

(40) Mecerreyes, D.; Lee, V.; Hawker, C. J.; Hedrick, J. L.; Wursch, A.; Volksen, W.; Magbitang, T.; Huang, E.; Miller, R. D. A Novel Approach to Functionalized Nanoparticles: Self-Crosslinking of Macromolecules in Ultradilute Solution. Adv. Mater. 2001, 13 (3), 204-208.

(41) Jiang, J.; Thayumanavan, S. Synthesis and Characterization of Amine-Functionalized Polystyrene Nanoparticles. Macromolecules 2005, 38 (14), 5886-5891. 
(42) de Luzuriaga, A. R.; Ormategui, N.; Grande, H. J.; Odriozola, I.; Pomposo, J. A.; Loinaz, I. Intramolecular Click Cycloaddition: An Efficient Room Temperature Route towards Bioconjugable Polymeric Nanoparticles. Macromol. Rapid Commun. 2008, 29 (12-13), $1156-$ 1160.

(43) de Luzuriaga, A. R.; Perez-Baena, I.; Montes, S.; Loinaz, I.; Odriozola, I.; García, I.; Pomposo, J. A. New Route to Polymeric Nanoparticles by Click Chemistry Using Bifunctional Cross-Linkers. Macromol. Symp. 2010, 296 (1), 303-310.

(44) Perez-Baena, I.; Asenjo-Sanz, I.; Arbe, A.; Moreno, A. J.; Lo Verso, F.; Colmenero, J.; Pomposo, J. A. Efficient Route to Compact Single-Chain Nanoparticles: Photoactivated Synthesis via Thiol-Yne Coupling Reaction. Macromolecules 2014, 47 (23), 8270-8280.

(45) Kröger, A. P. P.; Boonen, R. J. E. A.; Paulusse, J. M. J. WellDefined Single-Chain Polymer Nanoparticles via Thiol-Michael Addition. Polymer 2017, 120, 119-128.

(46) Sanchez-Sanchez, A.; Pérez-Baena, I.; Pomposo, J. A. Advances in Click Chemistry for Single-Chain Nanoparticle Construction. Molecules 2013, 18 (3), 3339-3355.

(47) Harth, E.; Van Horn, B.; Lee, V. Y.; Germack, D. S.; Gonzales, C. P.; Miller, R. D.; Hawker, C. J. A Facile Approach to Architecturally Defined Nanoparticles via Intramolecular Chain Collapse. J. Am. Chem. Soc. 2002, 124 (29), 8653-8660.

(48) Croce, T. A.; Hamilton, S. K.; Chen, M. L.; Muchalski, H.; Harth, E. Alternative O-Quinodimethane Cross-Linking Precursors for Intramolecular Chain Collapse Nanoparticles. Macromolecules 2007, 40 (17), 6028-6031.

(49) Altintas, O.; Willenbacher, J.; Wuest, K. N. R.; Oehlenschlaeger, K. K.; Krolla-Sidenstein, P.; Gliemann, H.; BarnerKowollik, C. A Mild and Efficient Approach to Functional SingleChain Polymeric Nanoparticles via Photoinduced Diels-Alder Ligation. Macromolecules 2013, 46 (20), 8092-8101.

(50) Hansell, C. F.; Lu, A.; Patterson, J. P.; O’Reilly, R. K. Exploiting the Tetrazine-norbornene Reaction for Single Polymer Chain Collapse. Nanoscale 2014, 6 (8), 4102-4107.

(51) Heiler, C.; Bastian, S.; Lederhose, P.; Blinco, J. P.; Blasco, E.; Barner-Kowollik, C. Folding Polymer Chains with Visible Light. Chem. Commun. 2018, 54 (28), 3476-3479.

(52) Hanlon, A. M.; Martin, I.; Bright, E. R.; Chouinard, J.; Rodriguez, K. J.; Patenotte, G. E.; Berda, E. B. Exploring Structural Effects in Single-Chain "Folding" Mediated by Intramolecular Thermal Diels-Alder Chemistry. Polym. Chem. 2017, 8 (34), $5120-5128$.

(53) He, J.; Tremblay, L.; Lacelle, S.; Zhao, Y. Preparation of Polymer Single Chain Nanoparticles Using Intramolecular Photodimerization of Coumarin. Soft Matter 2011, 7 (6), 2380-2386.

(54) Jiang, J.; Qi, B.; Lepage, M.; Zhao, Y. Polymer Micelles Stabilization on Demand through Reversible Photo-Cross-Linking. Macromolecules 2007, 40 (4), 790-792.

(55) Frank, P. G.; Tuten, B. T.; Prasher, A.; Chao, D.; Berda, E. B. Intra-Chain Photodimerization of Pendant Anthracene Units as an Efficient Route to Single-Chain Nanoparticle Fabrication. Macromol. Rapid Commun. 2014, 35 (2), 249-253.

(56) Frisch, H.; Menzel, J. P.; Bloesser, F. R.; Marschner, D. E.; Mundsinger, K.; Barner-Kowollik, C. Photochemistry in Confined Environments for Single Chain Nanoparticle Design. J. Am. Chem. Soc. 2018, 140 (30), 9551-9557.

(57) Trenor, S. R.; Shultz, A. R.; Love, B. J.; Long, T. E. Coumarins in Polymers: From Light Harvesting to Photo-Cross-Linkable Tissue Scaffolds. Chem. Rev. 2004, 104 (6), 3059-3077.

(58) Pomposo, J. A.; Rubio-Cervilla, J.; Moreno, A. J.; Lo Verso, F.; Bacova, P.; Arbe, A.; Colmenero, J. Folding Single Chains to SingleChain Nanoparticles via Reversible Interactions: What Size Reduction Can One Expect? Macromolecules 2017, 50 (4), 1732-1739.

(59) Smulders, M. M. J.; Buffeteau, T.; Cavagnat, D.; Wolffs, M.; Schenning, A. P. H. J.; Meijer, E. W. C3-Symmetrical Self-Assembled Structures Investigated by Vibrational Circular Dichroism. Chirality 2008, 20 (9), 1016-1022.
(60) Hosono, N.; Palmans, A. R. A.; Meijer, E. W. Soldier-SergeantSoldier" Triblock Copolymers: Revealing the Folded Structure of Single-Chain Polymeric Nanoparticles. Chem. Commun. 2014, 50 (59), 7990-7993.

(61) Nakano, Y.; Hirose, T.; Stals, P. J. M.; Meijer, E. W.; Palmans, A. R. A. Conformational Analysis of Supramolecular Polymerization Processes of Disc-like Molecules. Chem. Sci. 2012, 3 (1), 148-155.

(62) Gillissen, M. A. J.; Terashima, T.; Meijer, E. W.; Palmans, A. R. A.; Voets, I. K. Sticky Supramolecular Grafts Stretch Single Polymer Chains. Macromolecules 2013, 46 (10), 4120-4125. 